\title{
Pediatric Tuberculosis Disease during Years of High Refugee Arrivals: A 6-Year National Prospective Surveillance Study
}

\author{
Nora Fritschi ${ }^{a}$ Axel J. Schmidt ${ }^{b}$ Jürg Hammer ${ }^{c}$ Nicole Ritz $^{a, d, e}$ \\ Swiss pediatric surveillance unit \\ aDepartment of Clinical Research, Mycobacterial and Migrant Health Research Group, University Children's Hospital \\ Basel, University of Basel, Basel, Switzerland; ' Infectious Diseases Division, Swiss Federal Office of Public Health, \\ Bern, Switzerland; 'Division of Respiratory and Critical Care Medicine, University Children's Hospital Basel, University \\ of Basel, Basel, Switzerland; dInfectious Disease and Vaccinology Unit, University Children's Hospital Basel, \\ University of Basel, Basel, Switzerland; eDepartment of Pediatrics, The Royal Children's Hospital Melbourne, The \\ University of Melbourne, Parkville, VIC, Australia
}

\section{Keywords}

Tuberculosis · Asylum · Active tuberculosis · Surveillance study

\begin{abstract}
Background: In Europe, surveillance and monitoring of pediatric tuberculosis (TB) remains important, particularly in the light of migration in recent years. The aim of the study was to evaluate incidence rates of childhood TB and detailed diagnostic pathways and treatment. Methods: Data were collected through the Swiss Pediatric Surveillance Unit (SPSU) from December 2013 to November 2019. Monthly notifications are obtained from the 33 pediatric hospitals in the SPSU, and a detailed questionnaire was sent out upon notification. Inclusion criteria were children and adolescents aged up to 15 years with culture- or molecular-confirmed TB disease or for whom a treatment with $\geq 3$ antimycobacterial drugs had been initiated. Data were compared with agematched notification data from the Swiss Federal Office of Public Health (FOPH). Results: Of the 172 cases notified to SPSU, a detailed questionnaire was returned for 161 (93\%) children, of which 139 met the inclusion criteria. Reasons for
\end{abstract}

exclusion were age $>15$ years, double reporting, and not fulfilling the criteria for TB disease. During the same time period, 172 pediatric TB cases were reported to the $\mathrm{FOPH}$, resulting in an incidence of 2.1 per 100,000, ranging from 1.4 to 2.8 per year, without a clear trend over time. In the 64 (46.0\%) foreign-born children, incidence rates were higher and peaked in 2016, with 13.7 per 100,000 ( $p=0.018$ ). The median interval between arrival in Switzerland and TB diagnosis was 5 (IQR 1-21) months, and $80 \%$ were diagnosed within 24 months of arrival. In $58 \%$ of the cases, TB disease was confirmed by culture or molecular assays. Age $>10$ years, presence of fever, or weight loss were independent factors associated with confirmed TB. Conclusion: The annual pediatric TB incidence rate only varied among foreign-born children and was highest in 2016 when refugee influx peaked in Europe. Importantly, most foreign-born children with TB were diagnosed within 2 years after arrival in Switzerland. Thus, the early period after arrival in Switzerland is associated with a higher risk of TB disease in children, and this should be considered for screening guidance in refugees.

\section{(c) 2021 The Author(s)}

Published by S. Karger AG, Basel
(C) 2021 The Author(s)

Published by S. Karger AG, Basel

This is an Open Access article licensed under the Creative Commons Attribution-NonCommercial-4.0 International License (CC BY-NC) (http://www.karger.com/Services/OpenAccessLicense), applicable to the online version of the article only. Usage and distribution for commercial purposes requires written permission. 


\section{Introduction}

Tuberculosis (TB) disease remains a public health threat in Europe with an estimated 259,000 cases in the World Health Organization European region in 2018 [1]. In the same report, children $<15$ years account for $4 \%$ of all new and relapse TB cases in Europe, corresponding to an annual notification rate of 2.5/100,000.

Incidence rates are generally used to assess the burden of TB disease across countries but also across subpopulations. While the overall incidence of TB in Europe is stable on a low level, in recent years, higher incidence rates are observed in specific populations, including refugees in studies from Sweden [2], the UK [3], Italy [4], and the USA [5]. The rate of TB disease in children thereby serves as a sentinel for ongoing transmission of Mycobacterium tuberculosis as progression to TB disease in children occurs mostly within a short period of time after infection [6].

Migration has and continues to influence TB incidence in host countries worldwide [7, 8]. Refugees and asylum seekers have a higher risk for certain infectious diseases, including gastrointestinal infections, malaria, and TB $[9,10]$. Contributing factors for this increased risk are mainly the higher incidence of the disease in their country of origin and factors related to conditions during migration such as overcrowding, low hygiene standards, and malnutrition $[11,12]$.

Studies analyzing the impact of the most recent refugee crises on TB in Europe showed that up to 14\% of unaccompanied minor refugees had TB infection when screened in Germany [13] and Sweden in 2015 [14]. A Swiss study in newly arrived migrant children shows $7 \%$ of screened children had TB infection or TB disease, and an association with origin from a country with moderate to high TB incidence, older age, and contact to a TB index case was found [15]. However, detailed data on incidence rates, associated risk factors, and clinical characteristics in children with TB after migration to Europe are important and lacking in most national and regional reports $[16,17]$.

The aim of this study was to describe the incidence of TB disease in children notified to the Swiss Pediatric Surveillance Unit (SPSU) and the Swiss Federal Office of Public Health (FOPH) during the years of high refugee arrivals. Further to this, we aimed to characterize epidemiological factors, focusing on the origin and history of migration and analyze details of diagnostic pathways and treatment of TB in children.

\section{Methods}

Study Design and Population

Pediatric TB disease was actively monitored in a cross-sectional observational study as part of the SPSU from December 2013 to November 2019. All 33 national pediatric hospitals in Switzerland participated in the SPSU and notified the studied diseases in a monthly report (www.spsu.ch). Upon notification, a questionnaire was sent to the notifying hospital to obtain detailed clinical data (see online suppl. material; for all online suppl. material, see www.karger.com/doi/10.1159/000517029) by the local investigator. TB disease cases are also captured by the FOPH as TB is a mandatory notifiable disease. To ensure comparability between the 2 notification systems, we used the same case definition for the SPSU study as were in place for the FOPH notifications. A TB case was defined as the detection of $M$. $t u$ berculosis, M. africanum, M. bovis, $M$. caprae, or "Mycobacterium complex" by culture or molecular assays or when a treatment with $\geq 3$ antimycobacterial drugs was initiated. For the analysis of the pediatric cases, only individuals up to 15 years were included.

\section{Collected Variables}

The SPSU questionnaire (online suppl. material) included demographic characteristics such as age, sex, and country of birth of the child and parents, risk factors indicating exposure to an index case, clinical and radiologic findings, results of immunological, culture, and molecular diagnostics, and treatment parameters.

BCG vaccination status was assessed based on written documentation, characteristic scar, or history. Chest radiography findings and the use of further imaging including sonography, computed tomography $(\mathrm{CT})$, and magnetic resonance imaging were recorded.

Results of the tuberculin skin test (TST), T-SPOT.TB, and QuantiFERON-TB (QFT) tests were recorded as positive, negative, and borderline/indeterminate, and if available, with details of the diameter of induration, count of positive spots, and concentrations of interferon- $\gamma$. The following cutoffs determined a positive TST or interferon gamma release assay according to Swiss national recommendations and manufacturers' instructions [18]: a TST diameter of induration $>5 \mathrm{~mm}$, T-SPOT.TB number of spots in the TB antigen tubes $\geq 5$ spots, and QFT interferon $-\gamma$ concentration in the TB antigen tubes $\geq 0.35 \mathrm{IU}$.

Results of drug sensitivity testing were collected from culture or molecular-confirmed cases. Doses of all antimycobacterial and other drugs were recorded. From the variables notified to the $\mathrm{FOPH}$, the following were used in this study: demographic characteristics including age and sex and data from culture or molecular confirmation of TB diagnosis.

\section{Definitions}

For the SPSU and FOPH data set, we used 1-year periods, which lasted each from December to the following November and matched them to the population data for the following calendar year. The time point of study inclusion was defined by the month of notification for the SPSU data and by the month of diagnosis for the date from the FOPH. For the assessment of clinical and diagnostic findings, the study population was divided into 4 agegroups from 0 to $<2,2$ to $<5,5$ to $<10$, and 10 to 15 years of age [19]. 
Table 1. Baseline characteristics form cases notified to the SPSU and FOPH

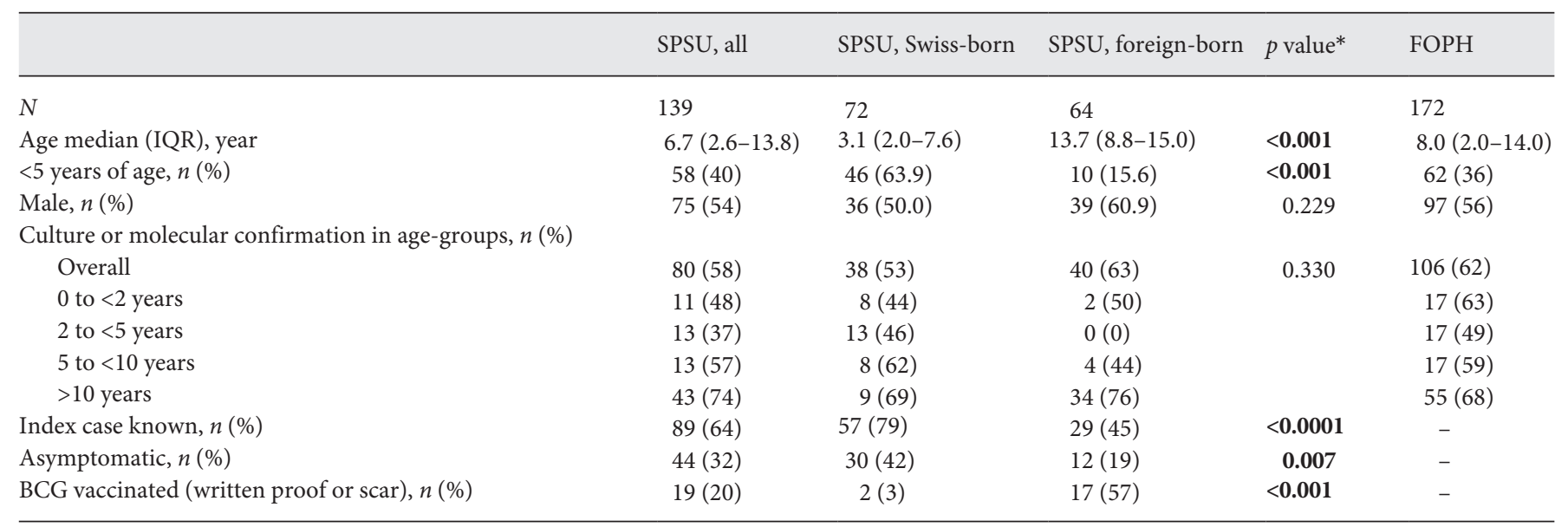

SPSU, Swiss Pediatric Surveillance Unit; FOPH, Swiss Federal Office of Public Health; IQR, interquartile range. Statistically significant $p$ values are in bold. *Swiss-born versus foreign-born.

\section{Statistical Analysis}

The population-based incidence was calculated using population data for individuals living in Switzerland aged up to 15 years provided by the Swiss Federal Statistical Office. Comparisons between Swiss-born and foreign-born children were done using the $\chi^{2}$ or Fisher exact test for categorical and the Wilcoxon rank sum test for non-normal distributed continuous variables. For the time since arrival in Switzerland in foreign-born children, the KaplanMeier estimate was used, and differences between groups were assessed with the log-rank test. The significance of the peak incidence among foreign-born children was assessed with a permutation test. Confidence intervals of incidence rates were calculated using exact Poisson distribution.

To identify clinical characteristics associated with culture and/ or molecular confirmation, univariable and multivariable logistic regression was done. A multivariable logistic regression model was fitted by including the variables origin and sex a priori and backward selection. A $p$ value of $<0.05$ was considered as potentially significant. Statistical analyses were done using $\mathrm{R}$ (version 4.0.0)/ $\mathrm{R}$ Studio (version 1.3.1073).

Ethics

The study was approved by the local Ethics Committee in March 2020 (Ethikkommission Nordwest-und Zentralschweiz, EKNZ 2020-000678).

\section{Results}

\section{Study Population}

Of the 172 cases notified to the SPSU, a detailed questionnaire was returned for 161 children with TB disease (94\%), and 139 were included in the final analysis. Reasons for exclusion were age $>15$ years $(n=12)$, double reporting $(n=8)$, and latent TB infection $(n=2)$. In comparison, 172 pediatric cases were notified to the FOPH in the same observational period, and these accounted for $5 \%$ of all notified TB disease cases in Switzerland. In children with TB disease notified to the SPSU, the median age was 6.7 (interquartile range [IQR] 2.6-13.8) years, and 58 ( $42 \%)$ children were $<5$ years of age. The median age of children notified to the FOPH was 8.0 (IQR 2.0-14.0), with $62(36 \%)$ children $<5$ years of age (Table 1$)$. In the SPSU data set, 72 children (52\%) were Swiss-born, 64 (46\%) were foreign-born, and for $3(2 \%)$ children, the country of birth was unknown (Fig. 1). Foreign-born children were from Eritrea (16\%), Somalia (9\%), and Afghanistan, Brazil, and Sudan ( $2 \%$ each). The median age of Swiss-born children was 3.1 years (IQR 2.0-7.6) and of foreign-born children 13.7 years (IQR 8.8-15.0) ( $p<$ $0.001)$. An index case as the source of infection was stated in $89(64.0 \%)$ children, and transmission occurred in $72 / 89(81 \%)$ children in the same household (online suppl. Table 1).

\section{Population-Based Incidence}

The overall incidence of TB disease in the SPSU and FOPH data set was 1.7/100,000 and 2.1/100,000, respectively. Overall incidence rates increased at the beginning of the observational period and peaked in 2016 at 2.2 (SPSU) and 2.8 (FOPH)/100,000 children in 2016 (Fig. 2). Incidence rates among Swiss-born children were consistently low during the observational period (0.4-1.3/100,000 children), while those of foreign-born children reached a significant peak in $2016(p=0.018)$ 
Fig. 1. Number of cases per age-group in all cases notified to the SPSU over the observational period stratified by country of birth of the children and their parents. SPSU, Swiss Pediatric Surveillance Unit.

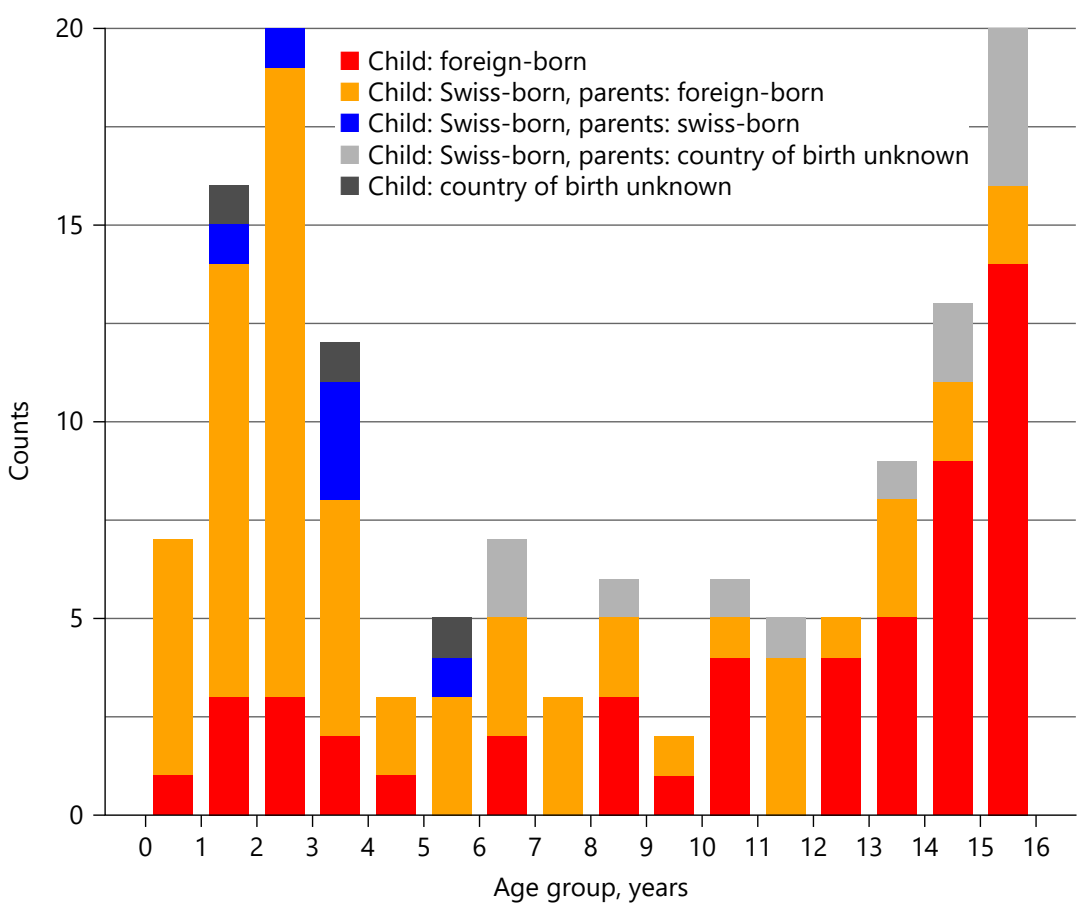

Fig. 2. Incidence of TB disease cases notified to the SPSU and FOPH over the observational period from 2014 to 2019. Incidence rate of foreign-born children were significantly higher than that in Swiss-born children and reached a maximum in 2016. SPSU, Swiss Pediatric Surveillance Unit; FOPH, Swiss Federal Office of Public Health; TB, tuberculosis.

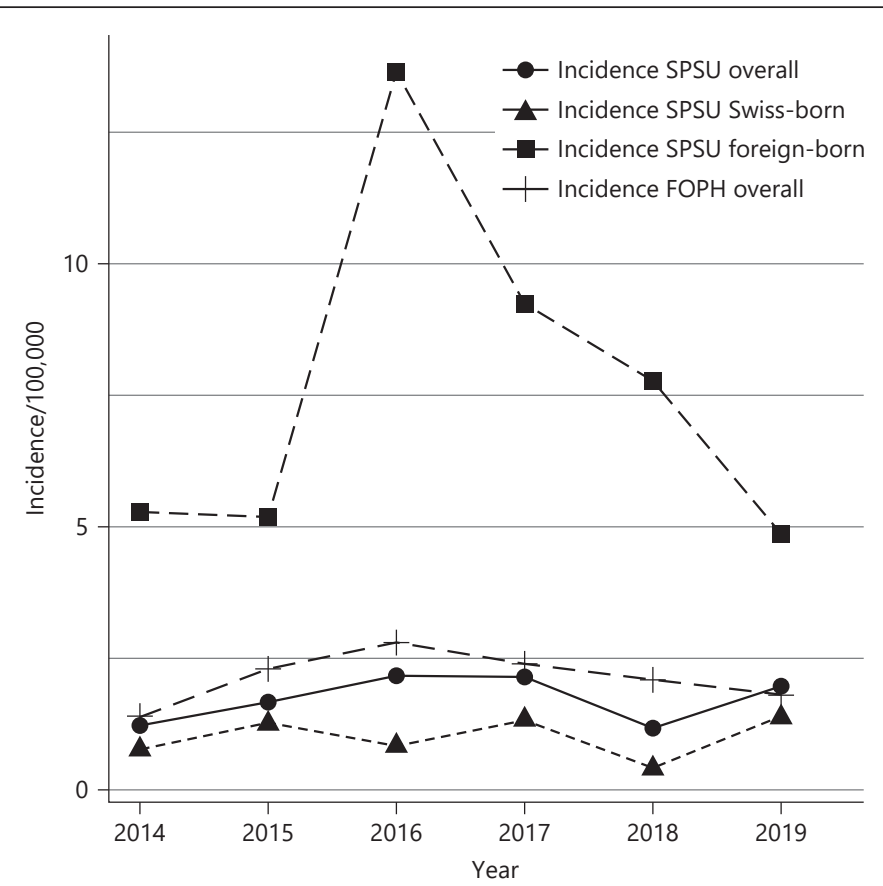

\begin{tabular}{|r|c|c|c|c|c|c|}
\hline$/ 100,000$ & 2014 & 2015 & 2016 & 2017 & 2018 & 2019 \\
\hline SPSU overall & 1.22 & 1.66 & 2.17 & 2.15 & 1.17 & 1.96 \\
\hline FOPH overall & 1.40 & 2.30 & 2.80 & 2.40 & 2.10 & 1.80 \\
\hline SPSU Swiss-born & 0.76 & 1.26 & 0.84 & 1.32 & 0.40 & 1.38 \\
\hline SPSU foreign-born & 5.28 & 5.19 & 13.70 & 9.25 & 7.78 & 4.87 \\
\hline
\end{tabular}

Pediatric Tuberculosis Disease during Years of High Refugee Arrivals 
Table 2. TB incidence in 2014-19 among different age-groups

\begin{tabular}{lll}
\hline Age-group & SPSU & FOPH \\
\hline$<2$ years & $2.23(95 \%$ CI $1.41-3.34)$ & $2.61(95 \%$ CI $1.72-3.80)$ \\
2 to $<5$ years & $2.26(95 \%$ CI $1.57-3.14)$ & $2.26(95 \%$ CI $1.57-3.14)$ \\
5 to $<10$ years & $0.91(95 \%$ CI $0.58-1.36)$ & $1.15(95 \%$ CI $0.77-1.65)$ \\
10 to $<16$ years & $1.97(95 \%$ CI $1.49-2.54)$ & $2.75(95 \%$ CI $2.18-3.42)$ \\
\hline
\end{tabular}

SPSU, Swiss Pediatric Surveillance Unit; FOPH, Swiss Federal Office of Public Health; TB, tuberculosis.

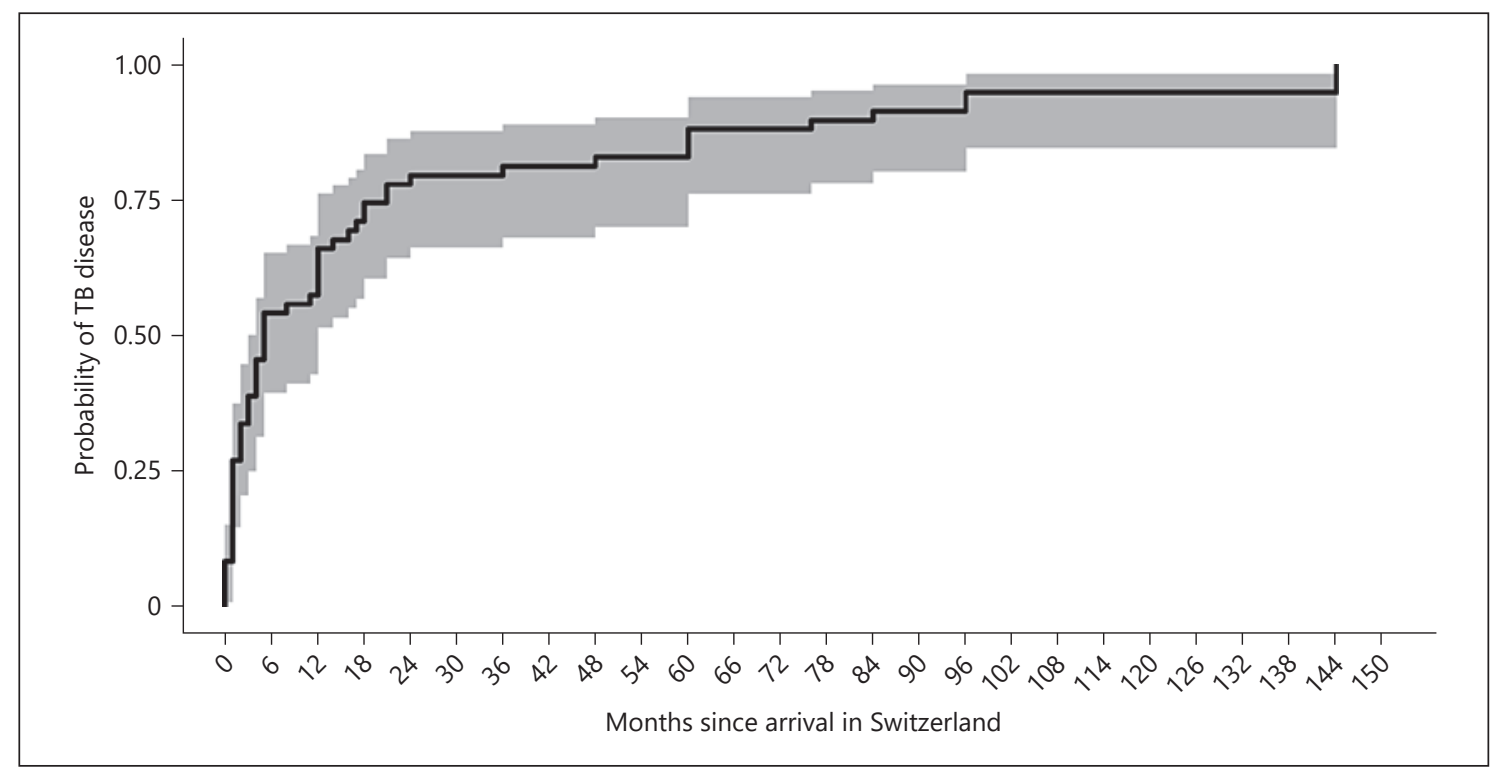

Fig. 3. Time analysis of foreign-born children displaying probability of TB disease diagnosis by time since arrival in Switzerland. Shaded area indicates 95\% CI. TB, tuberculosis; CI, confidence interval.

and were consistently $>1$ magnitude higher $(5.313 .7 / 100,000$ children, all $p$ values $<0.01)$. Children aged 5 to $<10$ years had the lowest incidence rate in both data sets (Table 2).

For the 67 foreign-born children, the time since arrival in Switzerland was recorded in $62(93 \%)$ cases. The median time from arrival to TB diagnosis was 5 (IQR $1-21)$ months. By 6 months, $54 \%$ of the children were diagnosed, and by 24 months, $80 \%$ of the children were diagnosed (Fig. 3). The longest time interval of TB diagnosis since arrival was 12 years, reported for 3 children; for 1 child, an exposure in the same household was stated, and for the other 2 the source of infection was unknown. The time interval between arrival in Switzerland to TB diagnosis was similar between agegroups $(<5,5$ to $<10$, and $>10$ years, $p=0.4)$ and similar in those with and without symptoms $(p=0.5)$, who were screened for TB upon arrival or investigated as part of contact tracing.

\section{Forms of TB and Clinical Presentation}

Detailed data on clinical presentation and risk factors were only available in the SPSU data set. A total of 118 children (85\%) had pulmonary TB. Of the 20 (14\%) children with extrapulmonary TB, the following affected areas were stated as follows: in 6 children, lymph node; in 5 , pleural; in 3, central nervous system; in 3, abdominal; in 2 cases, a miliary form without further specification; and missing information in 1 case. In the 95 (68\%) children with symptoms, the following were most common: cough in 63 (45\%), fever in 45 (32\%), weight loss in 44 (32\%), and wheezing in $5(4 \%)$ children. Cough was reported to be present for a median time of 28 (IQR 14-40) and fever with a median time of 12 (IQR 5-21) days. 
Table 3. Univariable and multivariable logistic regression analysis for factors associated with culture or molecular confirmation of TB disease

\begin{tabular}{llllllr}
\hline & \multicolumn{2}{l}{ Univariable log regression } & \multicolumn{3}{c}{ Multivariable log regression } \\
& OR & $95 \%$ CI & $p$ value & OR & 95\% CI & $p$ value \\
\hline Age 0 to $<$ 2 years & Reference & & \multicolumn{3}{c}{ Reference } \\
Age 2 to $<5$ years & 0.74 & $0.25-2.2$ & 0.592 & 0.78 & $0.24-2.61$ & 0.688 \\
Age 5 to $<10$ years & 1.44 & $0.44-4.81$ & 0.547 & 1.37 & $0.36-5.35$ & 0.643 \\
Age $>10$ years & 3.78 & $1.35-11.06$ & $\mathbf{0 . 0 1 3}$ & 4.40 & $1.23-17.32$ & 0.023 \\
Gender (male) & 1.07 & $0.53-2.14$ & 0.847 & 1.22 & $0.54-2.78$ & 0.629 \\
Origin (Swiss-born) & 0.61 & $0.30-1.23$ & 0.172 & 2.20 & $0.84-6.26$ & 0.121 \\
Asymptomatic & 0.21 & $0.09-0.45$ & $<\mathbf{0 . 0 0 0 1}$ & - & - & - \\
Cough & 1.63 & $0.81-3.31$ & 0.172 & - & - & - \\
Fever & 3.53 & $1.60-8.35$ & $<\mathbf{0 . 0 0 3}$ & 3.98 & $1.65-10.33$ & $\mathbf{0 . 0 0 3}$ \\
Weight loss & 5.98 & $2.52-15.66$ & $<\mathbf{0 . 0 0 1}$ & 4.19 & $1.51-12.84$ & $\mathbf{0 . 0 0 8}$ \\
\hline
\end{tabular}

The variable of presentation with or without symptoms was composed of variable reporting symptoms and was assessed only in the univariable models. For fitting the multivariable model, only variables of symptoms with sufficient observations were evaluated, and after backward selection, only fever and cough remained included. The grouped variable of presentation with or without symptoms was only assessed in the univariable model and not included in the multivariable model. Instead in multivariable model, the variables cough, fever, and weight loss were included, and variables for other symptoms were excluded for low number of recorded observations. Statistically significant $p$ values are in bold. TB, tuberculosis; OR, odds ratio; CI, confidence interval.

The Use and Results of Diagnostic Tests

In the SPSU data set, diagnosis was confirmed by culture or molecular techniques in $80(58 \%)$ children (Table 1). Of those, 73 (52\%) were positive by culture, 49 $(35 \%)$ by molecular assay, and $42(30 \%)$ by both methods. In 7 children, diagnosis was confirmed by molecular assay only, while culture remained negative, and in 34 children, diagnosis was confirmed by culture only, while molecular assay remained negative. The rate of confirmed TB was highest in children aged $>10$ years with $74.1 \%$. In the younger age-group, confirmation of TB was reported as follows: 5 to $<10$ years, $57 \%$; 0 to $<2$ years, $48 \%$; and 2 to $<5$ years, $37 \%$. The rate of confirmation was comparable in foreign-born (63\%) and Swiss-born children $(53 \%, p=0.33)$. Characteristics that were associated with TB confirmation in the univariable logistic regression were age, presence of fever, weight loss, and symptomatic presentation (Table 3 ). In a multivariable model, the age-group of children $>10$ years, the presence of fever, and the presence of weight loss remained independently associated with TB confirmation (Table 3).

Information on resistance testing from culture or molecular-confirmed cases was available in $68(85 \%)$ cases, of which 4 were isoniazid (two M. bovis infections, one $M$. tuberculosis, and one unknown isolate) and 3 isolates were pyrazinamide resistant, 2 of which were $M$. bovis infections. No multidrug resistant cases were reported.
Immuno-diagnostic test results were only available from the SPSU data set. The TST was done in $82(59 \%)$, T-SPOT.TB in 22 (16\%), and the QFT in $79(57 \%)$ cases and reported to be positive in 58 (71), 15 (68), and 60 (76\%) cases, respectively. In 7 children, the QFT showed an indeterminate result.

Chest radiography was performed for 135 (97.1\%) children and a chest CT scan in 52 (37.4\%). Neuroimaging with $\mathrm{CT}$ scan or magnetic resonance imaging was done in $10(7.2 \%)$ children. Ultrasound was performed in $32(23 \%)$ children, mostly for abdominal imaging (in 22, $69 \%)$.

On chest radiography, hilar lymphadenopathy and consolidation were the most frequently found abnormalities. Bronchial compression was found more frequently in children $<2$ years, calcified lymph nodes more frequently in children 5 to $<10$ years, and effusion and caverns more frequently in the children $>10$ years old (Fig. 4).

A total of 78 children (56\%) were tested for HIV infection, all of which were negative. Swiss-born children were significantly less often tested (in 28 cases, $39 \%$ ) than foreign-born children (in 43 cases, $82 \%$; $p$ value $<0.0001$ ).

\section{Choice and Dosing of Antituberculous Medication}

Details on treatment were only available in the SPSU data set. Overall, 69 (49.6\%) children received a triple (isoniazid, rifampicin, and pyrazinamide) and 53 (37.4\%) 
a quadruple treatment (with the addition of ethambutol). The range of drug dosages prescribed was highly correlated with the national guidelines; some children received fixed dose combinations (online suppl. Fig. 1).

\section{Discussion}

Since our last detailed analysis of childhood TB notifications in Switzerland for the years 1996-2011, this is the first analysis including a period of high migrant arrivals in Switzerland [20]. The current overall annual TB incidence rates of 1.7-2.1/100,000 children in the years 20142019 are slightly higher than those in the earlier time period, which was 1.6/100,000 children. Of particular interest is the considerable increase of the TB incidence rate in 2016 and the higher incidence in foreign-born children. This has been highly likely influenced by the large migrant arrival of refugees and asylum-seeking children in all European countries in 2015 and 2016. Similar to our findings, higher TB incidence rates among foreign-born than those in native-born children were observed in other European countries, like Sweden [21], the Netherlands [22], and the UK [3]. Similarly, a prospective study in the region of Campania in Italy also observed increasing $\mathrm{TB}$ incidence rates among children over an observational period from 2009 to 2018 [23]. The authors of that study observed a correlation between TB incidence and the rate of migrants living in the region, suggesting that the disease occurrence was highly likely influenced by the influx of migrants.

\section{Time to Diagnosis since Arrival}

We found that two-thirds of the cases in foreign-born children are diagnosed within 1 year of arrival in Switzerland. The finding that TB disease in refugees and asylum seekers manifests early after arrival in the host country has been described in other countries. For example, studies from the Netherlands and Israel found that $53-58 \%$ of migrant children developed TB disease within the first year after arrival [24, 25]. A further study in the USA showed that time from arrival to TB diagnosis was highly variable and influenced by the country of origin [5]. A shorter time to TB diagnosis with a mean of 1.2 years was seen for children originating from China, Burma, and Ethiopia, whereas as longer intervals with a mean of 4.5 years were seen for children originating from Mexico [5].

A large proportion of foreign-born children originated from non-European countries and therefore had long migration routes. Factors related to the migration, like over-

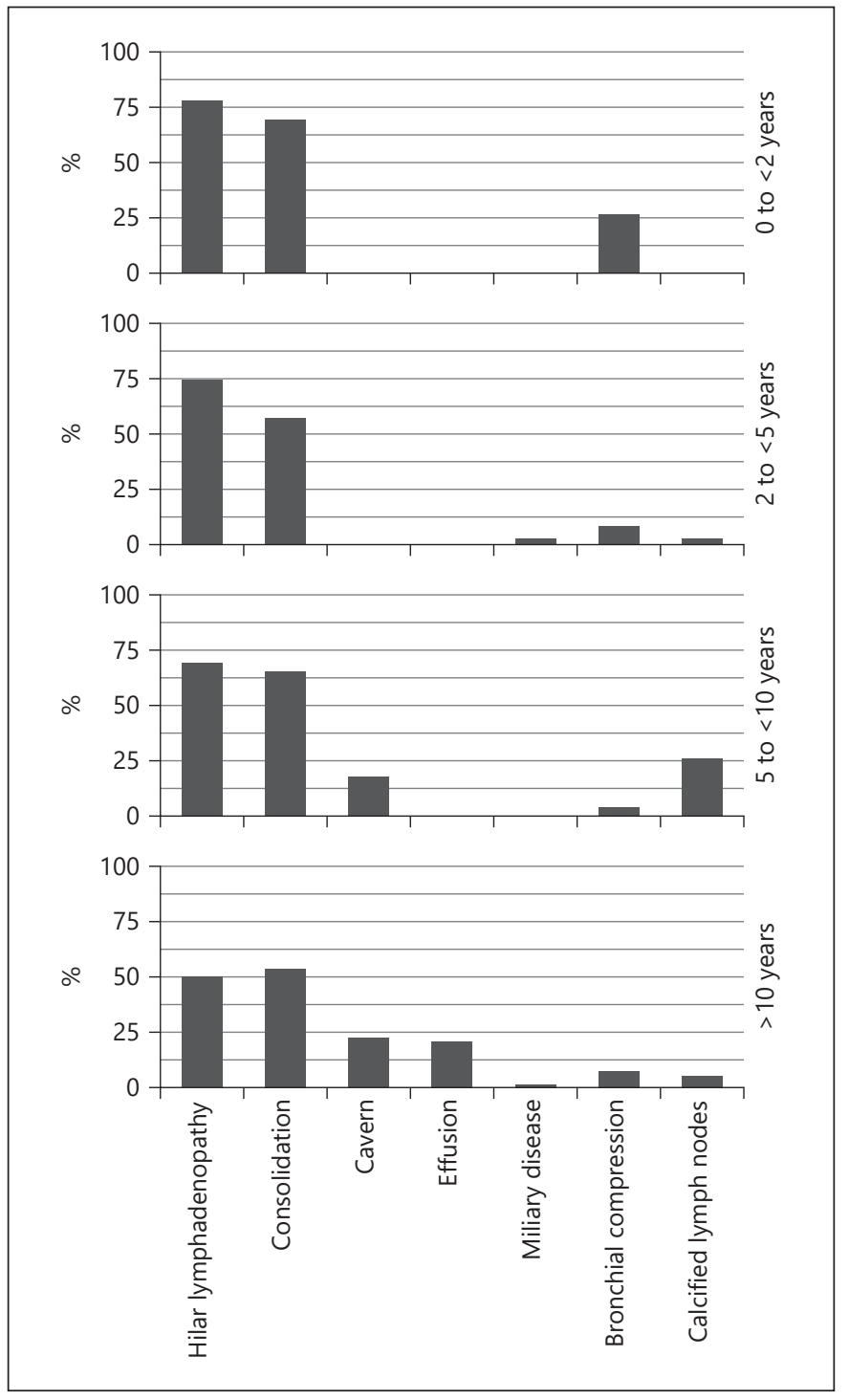

Fig. 4. Findings on chest radiography by age-group.

crowding and malnutrition, are assumed to contribute to the higher risk of TB disease in the initial period after arrival [26]. In addition, these factors could also contribute to the progression to TB disease in children with previously acquired TB infection. For children who develop TB years after arrival in the host country, it remains unclear if they are newly infected in the host country or have taken longer to progress to TB disease. Our study indicates that foreign-born children are at particular risk early after arrival, which should be considered for early screening guidance. The fact that a third of foreign-born children had TB diagnosed $>1$ year after arrival underlines the importance of regular health checks of refugee 
and asylum-seeking children. Screening for TB in migrants remains therefore important, and cost effectivity has been shown in our setting $[27,28]$.

\section{Culture Confirmation}

In our study, the ratio of children with culture or molecular confirmation of TB was approximately $60 \%$. This is higher, than what we have found in our previous analysis and also what is commonly reported in other European countries, where culture confirmation reaches approximately $50 \%[20,29]$. In addition, in a high-burden TB setting, culture confirmation is even lower with a median proportion of $29 \%$ as shown by a meta-analysis [30]. We found that age, presence of fever, and weight loss were independently associated with culture and/or molecular confirmation. A recently published study from the UK showed that older, female, foreign-born children, and those with pulmonary disease are more likely to be culture or molecular confirmed [3]. Similarly, in our study, children aged $>10$ years were more likely to be culture or molecular confirmed, whereas no effect of sex and country of origin was observed in both univariable and multivariable models. Weight loss is a symptom commonly seen late in the TB disease course; therefore, it seems reasonable that we observed an association with culture or molecular confirmation. In contrast, the finding that the presence of fever is associated with culture or molecular confirmation was somehow surprising as it is assumed to appear rather early in disease course, when TB might still be paucibacillary, and we would not expect confirmation of the diagnosis $[31,32]$.

\section{Radiologic Imaging}

Radiologic imaging with chest radiography is a standard investigation when pulmonary TB is suspected [33, 34]. Hilar lymphadenopathy and consolidation were most frequently observed in all age-groups [35]. Despite the small number of included children, bronchial compression was most observed in children aged $<2$ years, which is a typical finding in this age-group known from the prechemotherapy era [6]. Caverns and effusion are characteristics of adult-type TB and were also most often observed in the oldest age-group in our study. In contrast to studies from the prechemotherapy era, in which caverns were mostly observed in children older than 10 years, we also observed caverns in younger children [6]. The significance of caverns in younger children is less clearly understood; several case series have described caverns in younger children, and it was suggested that distinct pathophysiological mechanisms such as bronchial ob- struction and liquification of primary focus contribute to the development of caverns in this age-group [36].

$\mathrm{CT}$ is recommended for the diagnosis of TB in children with inconclusive clinical presentation and diagnostics or to search for local complications. The use of CT imaging in about $40 \%$ of cases in our study was remarkable, but in a comparable range to an Italian study of $>500$ children with active TB from 2010 to 2012, CT was also used in a high proportion of 56\% [4]. Despite not having collected detailed data on the indications for CT, we assume that in most cases, it was used to rule out an alternative diagnosis and local complications. CT is indicated in specific circumstances, but its indication should be carefully evaluated as it affords an exposure to radiation, application of contrast, and sedation in young children. Alternative imaging methods, like sonography, where radiation could be avoided, should be also strengthened in the evaluation of pulmonary TB.

\section{Limitations}

Our study has several limitations. The observed annual incidence rates were consistently lower in the SPSU than those in the FOPH notification data, suggesting that the mandatory system routinely captures more cases. A direct patient-to-patient comparison of the children in the 2 data sets was not possible, which prevents an estimation of the notification rate by a capture-recapture method. The observational character of our study and the restriction to children with TB disease prevents an analysis of risk factors for progression of the disease. Despite having collected detailed data on treatment, our study did not systematically include adverse effects of treatment, changes in treatment regimen, and outcome measurement, such as completion of treatment or TB relapse rates. However, none of the children were notified to have been treated for TB before inclusion in this study.

\section{Conclusion}

The complementary character of the 2 notification systems used in the study enables further insights of TB epidemiology in children. The study shows that annual TB incidence rates varied significantly between foreignborn and Swiss-born children and were timely related to the years with high refugee arrival. In particular, the early period after arrival in Switzerland is associated with a higher risk of TB disease in children, and this should be 
considered for screening guidance in refugees. Continuous monitoring of incidence rates in children and refugees are important and may help to identify early trends of TB epidemiology in the general population.

\section{Acknowledgements}

We thank the representatives of the pediatric units in Switzerland: M. Albisetti, W. Bär, V. Bernet, M. Bianchetti, H.U. Bucher, L. Buetti, M. Büttcher, F. Cachat, A. Castiglione, V. Colombo, C. Däster, P. Diebold, Z. Dovhunovà, G. Duvoisin, S. Ferroni, S. Fluri, M. Gebauer, M. Gehri, E. Giannoni, S. Grupe, L. Hegi, K. HeldEgli, M. Horn, P. Imahorn, T. Karen, T. Keller, C. Kind, L. Kottanattu, B. Laubscher, U. Lips, H. Madlon, V. Maghaouri-Slim, A. Malzacher, J. Mc Dougall, J.-C. Minet, M. Mönkhoff, A. Moser, A. Niederer, V. Muehlethaler, A. Niederer, V. Pezzoli, N. Piol, K. Posfay Barbe, G. Ramos y Munoz, L. Reinhard, T. Riedel, H. Roten, C. Rudin, K.P. Rühs, M. Russo, P. Schillinger, V. Schlumbom, N. Schöbi, G. Simonetti, S. Stirnemann, C. Stüssi, E. Süess, R. Tabin, M. Tomaske, A. Ughetto, R. Villiger, S. Wellmann, J. Wildhaber, K. Woll, M. Wopmann, G. Zeilinger, A. Zemmouri, and S.-A. Zoubir.

\section{Statement of Ethics}

The study was approved by the local Ethics Committee in March 2020 (Ethikkommission Nordwest-und Zentralschweiz, EKNZ 2020-000678). This study was done in accordance with Article 34 of the Swiss Human Research Act, which allows the further use of anonymized personal health-related data under the waiver of informed consent.

\section{Conflict of Interest Statement}

The authors declare that they have no known competing financial interests or personal relationships that could have appeared to influence the work reported in this paper.

\section{Funding Sources}

There was no funding source for the realization of this study.

\section{Author Contributions}

This study was designed by N.R. and J.H.; data analysis was done by N.F. and A.J.S.; N.F. and N.R. wrote the first draft; all the authors revised the manuscript and approved the final draft.

\section{References}

1 European Centre for Disease Prevention and Control. Tuberculosis surveillance and monitoring in Europe 2020. 2020.

2 Bennet R, Nejat S, Eriksson M. Foreign background and older age of children were associated with infection in Swedish tuberculosis contacts. Acta paediatr. 2020;109(9):1854-9.

3 Mohiyuddin T, Seddon JA, Thomas HL, Lalor MK. The changing landscape of childhood tuberculosis in the United Kingdom: a retrospective cohort (2000-2015). Pediatr Infect Dis J. 2019;38(5):470-5.

4 Galli L, Lancella L, Tersigni C, Venturini E, Chiappini E, Bergamini BM, et al. Pediatric tuberculosis in Italian children: epidemiological and clinical data from the Italian register of pediatric tuberculosis. Int J Mol Sci. 2016; 17(6):960.

5 Winston CA, Menzies HJ. Pediatric and adolescent tuberculosis in the United States, 2008-2010. Pediatrics. 2012;130(6):e1425-32.

6 Marais BJ, Gie RP, Schaaf HS, Hesseling AC, Obihara CC, Starke JJ, et al. The natural history of childhood intra-thoracic tuberculosis: a critical review of literature from the pre-chemotherapy era. Int J Tuberc Lung Dis. 2004; 8(4):392-402.

7 Proença R, Mattos Souza F, Lisboa Bastos M, Caetano R, Braga JU, Faerstein E, et al. Active and latent tuberculosis in refugees and asylum seekers: a systematic review and meta-analysis. BMC Public Health. 2020;20(1): 838.
8 Kimbrough W, Saliba V, Dahab M, Haskew C, Checchi F. The burden of tuberculosis in crisis-affected populations: a systematic review. Lancet Infect Dis. 2012;12(12):950-65.

9 Gmünder M, Brandenberger J, Buser S, Pohl C, Ritz N. Reasons for admission in asylumseeking and non-asylum-seeking patients in a paediatric tertiary care centre. Swiss Med Wkly. 2020;150:w20252.

10 Pohl C, Mack I, Schmitz T, Ritz N. The spectrum of care for pediatric refugees and asylum seekers at a tertiary health care facility in Switzerland in 2015. Eur J Pediatr. 2017;176(12):1681-7.

11 Hayward S, Harding RM, McShane H, Tanner R. Factors influencing the higher incidence of tuberculosis among migrants and ethnic minorities in the UK. F1000Res. 2018;7:461.

12 Brandenberger J, Tylleskär T, Sontag K, Peterhans B, Ritz N. A systematic literature review of reported challenges in health care delivery to migrants and refugees in high-income countries: the $3 \mathrm{C}$ model. $\mathrm{BMC}$ public health. 2019;19(1):755.

13 Thee S, Krüger R, von Bernuth H, Meisel C, Kölsch U, Kirchberger V, et al. Screening and treatment for tuberculosis in a cohort of unaccompanied minor refugees in Berlin, Germany. PLoS One. 2019;14(5):e0216234.

14 Bennet R, Eriksson M. Tuberculosis infection and disease in the 2015 cohort of unaccompanied minors seeking asylum in Northern Stockholm, Sweden. Infect Dis (Lond). 2017; 49(7):501-6.
15 Boukamel M, Fougère Y, Gehri M, Suris JC, Rochat I, Miletto D, et al. Prevalence of tuberculosis in migrant children in Switzerland and relevance of current screening guidelines. Swiss Med Wkly. 2020;150:w20253.

16 Gröschel MI, van den Boom M, Migliori GB, Dara M. Prioritising children and adolescents in the tuberculosis response of the WHO European Region. Eur Respir Rev. 2019;28(151).

17 Straetemans M, Bakker MI, Alba S, Mergenthaler C, Rood E, Andersen PH, et al. Completeness of tuberculosis (TB) notification: inventory studies and capture-recapture analyses, six European Union countries, 2014 to 2016. Euro Surveill. 2020;25(12).

18 Schweiz L. Manual of tuberculosis: revised version January 2021. 2021.

19 Cuevas LE, Browning R, Bossuyt P, Casenghi M, Cotton MF, Cruz AT, et al. Evaluation of tuberculosis diagnostics in children: 2. Methodological issues for conducting and reporting research evaluations of tuberculosis diagnostics for intrathoracic tuberculosis in children. Consensus from an expert panel. J Infect Dis. 2012;205 Suppl 2:S209-15.

20 Oesch Nemeth G, Nemeth J, Altpeter E, Ritz N. Epidemiology of childhood tuberculosis in Switzerland between 1996 and 2011. Eur J Pediatr. 2014;173(4):457-62.

21 Nejat S, Buxbaum C, Eriksson M, Pergert M, Bennet R. Pediatric tuberculosis in Stockholm: a mirror to the world. Pediatr Infect Dis J. 2012;31(3):224-7. 
22 Erkens CG, de Vries G, Keizer ST, Slump E, van den Hof S. The epidemiology of childhood tuberculosis in the Netherlands: still room for prevention. BMC Infect Dis. 2014; 14:295.

23 Lo Vecchio A, Smarrazzo A, Amato C, Palladino R, Scarano SM, Spagnuolo MI, et al. Increasing tuberculosis rates and association with migration in children living in Campania region, Southern Italy: a 10-year cohort study. Pediatr Infect Dis J. 2020;39(11):101722.

24 Nordholm AC, Holden IK, Hartling UB, Andersen PH, Lillbaek T, Johansen IS. The epidemiology and clinical characteristics of pediatric tuberculosis in Denmark 2009-2014 highlights targets for prevention. Pediatr Infect Dis J. 2020;39(11):1007-11.

25 Mor Z, Cedar N, Pinsker G, Bibi H, Grotto I. Childhood tuberculosis in Israel: epidemiological trends and treatment outcomes, 19992010. Eur Respir J. 2013;41(5):1157-62.
26 Baauw A, Kist-van Holthe J, Slattery B, Heymans $M$, Chinapaw M, van Goudoever $\mathrm{H}$. Health needs of refugee children identified on arrival in reception countries: a systematic review and meta-analysis. BMJ Paediatr Open. 2019;3(1):e000516.

27 Usemann J, Ledergerber M, Fink G, Ritz N. Cost-effectiveness of tuberculosis screening for migrant children in a low-incidence country. Int J Tuberc Lung Dis. 2019;23(5):579-86.

28 Ritz N, Brinkmann F, Santiago Garcia B, Tebruegge M, Kampmann B; Paediatric Tuberculosis Network European Trials Group. Tuberculosis in young refugees. Lancet. 2015; 386(10012):2475-6.

29 Ritz N, Curtis N. Novel concepts in the epidemiology, diagnosis and prevention of childhood tuberculosis. Swiss Med Wkly. 2014; 144:w14000.

30 DiNardo AR, Detjen A, Ustero P, Ngo K, Bacha J, Mandalakas AM. Culture is an imperfect and heterogeneous reference standard in pediatric tuberculosis. Tuberculosis. 2016; 101:S105-8.
31 Tebruegge M, Ritz N, Curtis N, Shingadia D. Diagnostic tests for childhood tuberculosis: past imperfect, present tense and future perfect? Pediatr Infect Dis J. 2015;34(9):1014.

32 Nicol MP, Zar HJ. Advances in the diagnosis of pulmonary tuberculosis in children. Paediatr Respir Rev. 2020;36:52-6.

33 Perez-Velez CM, Marais BJ. Tuberculosis in children. N Engl J Med. 2012;367(4):348-61.

34 Gwee A, Pantazidou A, Ritz N, Tebruegge M, Connell TG, Cain T, et al. To x-ray or not to $\mathrm{x}$-ray? Screening asymptomatic children for pulmonary TB: a retrospective audit. Arch Dis Child. 2013;98(6):401-4.

35 Naranje P, Bhalla AS, Sherwani P. Chest tuberculosis in children. Indian J Pediatr. 2019; 86(5):448-58.

36 Griffith-Richards SB, Goussard P, Andronikou S, Gie RP, Przybojewski SJ, Strachan M, et al. Cavitating pulmonary tuberculosis in children: correlating radiology with pathogenesis. Pediatr Radiol. 2007;37(8):798-9; quiz 48-9. 